\title{
ISOLATION OF AEROMONAS SPECIES FROM DIARRHEAL PATIENTS IN ISMAILIA GOVERNORATE
}

Samar M. Mansour ${ }^{1}$, Salah M. Abdalla ${ }^{1 *}$, Mohamed Farid A. F. Ali ${ }^{2}$ and Alaa A. Zeitoun ${ }^{3}$

${ }^{1}$ Department of Microbiology and Immunology, Faculty of Pharmacy, Suez Canal University, Ismailia, Egypt

${ }^{2}$ Department of Microbiology and Immunology, Faculty of Medicine, Suez Canal University, Ismailia, Egypt

${ }^{3}$ Department of Pediatrics, Faculty of Medicine, Suez Canal University, Ismailia, Egypt

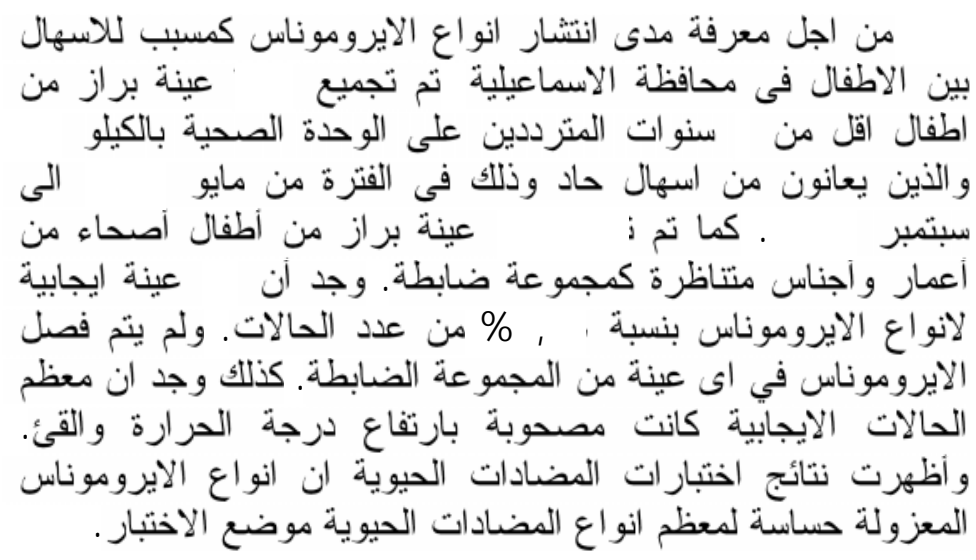

In order to know the prevalence of Aeromonas species as a causative agent of diarrhea in Ismailia, Three hundreds and fifty stool samples and rectal swabs were collected from infants and children under the age of 5 years visiting El Kilo11Clinic suffering from acute diarrhea in the period from May 2004 to September 2004. A stool samples were collected from control group of fifty healthy infants and children of matched age and sex. It was found that 29 diarrheal samples were positive for Aeromonas species with prevalence of $8.28 \%$. None of control samples were positive for Aeromonas. In most cases it was found that the infection is water or

Received in 12/9/2005 \& Accepted in 31/10/2005

"Corresponding author 
food borne. Aeromonas strains show high degree of antibiotic susceptibility.

\section{INTRODUCTION}

The genus Aeromonas is a gram negative oxidase positive micro organism and it was a member of the family Vibrionaceae which included vibrio and Plesiomonas species. ${ }^{1}$ Its separation from the genera Vibrio and Plesiomonas depends on resistance to the vibriostatic compound $\mathrm{O} / 129$, no growth on sodium chloride and absence of Ornithine decarboxylase except in A. veronii biovar veronii. ${ }^{1}$ Now it creates a new family Aeromonaceae.

Some strains of Aeromonas bacteria (aeromonads) are a significant, yet often underrated, cause of gastroenteritis, particularly in children under 5 years old and older persons in the summer. ${ }^{2 \& 3}$ They can also cause life-threatening infections, such as septicemia and meningitis in immunocompromized individuals. ${ }^{4 \& 5}$

Aeromonas are widely distributed in nature and can cause diseases in fish, reptiles, and amphibians. ${ }^{6}$

The Aeromonas species have been isolated from natural mineral water and well water. ${ }^{7}$

In one study in Chennai, two hundered stool samples were taken from children younger than 10 year old having diarrhea, Aeromonas was isolated from $6.5 \%$ of samples. ${ }^{8}$

In another study in Bangladesh Aeromonas was isolated from 5.5\% of cases of acute diarrhea among children. $^{9}$ And of 130 diarrheic patients, Aeromonas species were isolated in nine patients $(6.9 \%)$ in Hong Kong as reported by Siu-Wa and Cheung. ${ }^{10}$

Aeromonas species involved in travellers diarrhea as reported by Vila, et $a l^{11}$ who determined the prevalence of Aeromonas species associated with travelers diarrhea and found that Aeromonas species were isolated from $(2 \%)$ of travelers in Spain.

There are various subspecies, including Aeromonas Salmonicida, a fish pathogen, ${ }^{6}$ and Aeromonas hydrophila, a human pathogen. Approximately $85 \%$ of diarrheaassociated with Aeromonas, isolates belong to the species Aeromonas hydrophila (HG1 and HG3), A. veronii biovar sobria (HG8/10) (formerly A. sobria), and A. caviae (HG4). ${ }^{1}$ However, it is not yet possible to identify virulent strains definitively. The alt and act genes, which encode heat-labile cytotonic and cytotoxic enterotoxins respectively, seem to be related strongly to the diarrhea associated with Aeromonas and they found in 71.9 and $20.1 \%$ of strains respectively, examined in one study. ${ }^{3}$ The infection is strongly associated with drinking untreated water. ${ }^{7}$

Patients usually presented with a spectrum of diarrhea from mild to 
sever abdominal cramps, vomiting, and fever. Blood in stool can be seen as well. ${ }^{12}$ In some cases the symptoms are sever enough to required intravenous fluid therapy and observation and persistant diarrhea is uncommon as reported by Siu-Wa and Cheung. ${ }^{10}$

The highest proportion of Aeromonas diarrhea was found in younger children, particularly in those under 3 years of age. ${ }^{9}$

Flouroquinolone are the drug of choice for the treatment of Aeromonas induced infection but it should not be used in treating pediatric patients. ${ }^{13}$ Alternative therapies include trimethoprimsulfamethoxazole, aminogycosides, imipenem, meropenem, parenteral broad spectrum cephalosporins, and tetracyclines. ${ }^{14 \& 15}$

The main objective of this study is to determine the prevalence of Aeromonas species as a diarrheal cause among children in Ismailia and their antibiotic susceptibility.

\section{MATERIALS AND METHODS}

\section{Patients}

350 infants and children under the age of 5 years with the following criteria:

1- Acute diarrhea (defined as three or more stools a day and lasting less than 14 days).

2- Bloody or watery diarrhea.

3- Children not received antibiotic 2 days before sample collection.

4- May have one of the following symptoms or all: fever, vomiting, and abdominal cramps.
Control group of 50 healthy infants and children of matched age and sex. Control group had not taken antibiotic during the previous 2 weeks of samples collection.

\section{Microbiological media}

1- Alkaline peptone water PH 8.6.

2- Ampicillin blood agar (30 microgram/liter).

3- Cefzolidine-Irgasan-Novobiocine (CIN) agar.

4- Aeromonas selective agar.

5- Muller Hinton agar.

\section{Microbiological tests}

Stool samples were enriched on alkaline peptone water overnight at $37^{\circ}$ and were streaked on Ampicillin blood agar, CIN agar and Aeromonas selective agar for 24 hour at $37^{\circ}$. Presumptive Aeromonas isolates were screened for standard phenotypic traits ( $\beta$-haemolysis on blood agar, oxidase positive and indol positive) and species identity was determined using API-20E identification system.

\section{Antibiotic Susceptibility}

Antibiotic susceptibility patterns were determined by the disk diffusion method on Muller Hinton agar using this antibiotic which supplied from

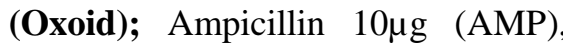

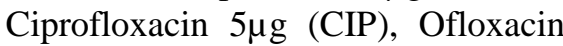
$10 \mu \mathrm{g}$ (OFX), Chloramphenicol $30 \mu \mathrm{g}$ (C), Cefatriaxone $30 \mu \mathrm{g}$ (CRO), Cefepime $30 \mu \mathrm{g}$ (FEP), Sulfamethoxazole / Trimethoprim $25 \mu \mathrm{g}$ (SXT). The susceptibility of the strains was determined according to the size of inhibition zone. ${ }^{16}$ 


\section{RESULTS AND DISCUSSION}

\section{Culture and isolation}

Culture for Aeromonas species were performed on 350 diarrheic stool specimens. Twenty nine samples were found to be positive for Aeromonas species of them 8 strains identified as A. hydrophila, 8 strains identified as A. caviae, and 13 strains identified as $A$. sobria. None of the control group samples was positive to Aeromonas species. The isolation rate in this study was $8.28 \%$ among infants and children visiting El Kilo 11 Clinic in Ismailia Governorate as shown in Table 1.

Isolation rates of Aeromonas from diarrheal stool vary, ranging from a low rate of $0.7-0.9 \%$ in the Netherlands and France, to medium rate of $2.5-7.1 \%$ in the United States, $5.5 \%$ Among Bangladeshi children, 6.5\% in Chennai, $11 \%$ in Japan, $24 \%$ in Ivory Coast, and to a high rate of $50 \%$ in infants in Peru as reported by Komathi et al. ${ }^{8}$ Teka et $a l .{ }^{9}$ and Pazzaglia, et al. ${ }^{17}$

In agreement with Holemberg et $a l^{2}$ and Albert et al..$^{3}$ the median age of the patients' population was 19 months (range from 3 months to 5 years). Of whom $59 \%$ females and $41 \%$ males.

Most of Aeromonas associated gastroenteritis is food or water borne. The clinical symptoms in Aeromonasassociated diarrhea were fever in 26 cases out of 29 positive cases $(89.6 \%)$, vomiting in 18 cases out of 29 positive cases $(62 \%)$, abdominal cramps in 3 cases out of 29 positive cases $(10.3 \%)$, and bloody diarrhea were seen in 2 cases only (6.9\%) as shown in Figure 1. Numbers of attacks of diarrhea per day were varied among cases between 2 times to 20 times.

The distribution of Aeromonas cases on summer months were as follow: May, 2 cases out of 51 cases (3.9\%), June, 5 cases out of 86 cases (5.8\%), July, 10 cases out of 94 cases (10.6\%), August, 7 cases out of 67 cases (10.4\%), and September, 5 cases out of 52 cases $(9.6 \%)$ as shown in Figure 2. It was noticed that Aeromonas-associated diarrhea was most common in the peak of July to September, this may be explained by the peak of hot humid weather.

\section{Antibiotic susceptibility results}

The antibiotic susceptibility of the isolated Aeromonas strains to ciprofloxacin and ofloxacin was $96.6 \%$. Susceptibility to cefepime and cefatriaxone was $93.1 \%$ and $93.1 \%$ respectively as shown in Figure 3.

The susceptibility to chloramphenicol and sulfamethoxazole was $63 \%$ and $55.5 \%$ respectively. One strain of Aeromonas was sensitive to ampicillin.

Aeromonas showed a high degree of antibiotic susceptibility that agreed with previous reports. ${ }^{10,13 \& 15}$

The susceptibility of chloramphenicol and sulfamethoxazoletrimethoprim was $63 \%$ and $55.5 \%$ that indicate increase the resistance to these antibiotic that agreed with Ko et $a l .{ }^{14}$ Broad spectrum cephalosporins such as cefepime and cefatriaxone 
Table 1: Prevalence of Aeromonas among diarrheal samples and control samples.

\begin{tabular}{||l|c|c|c|c|c||}
\hline & $\begin{array}{c}\text { Positive } \\
\text { cases }\end{array}$ & Percentage & $\begin{array}{c}\text { Negative } \\
\text { cases }\end{array}$ & Percentage & Total \\
\hline Diarrheal samples & 29 & $8.28 \%$ & 321 & $91.72 \%$ & 350 \\
\hline Control samples & 0 & $0 \%$ & 50 & $100 \%$ & 50 \\
\hline
\end{tabular}

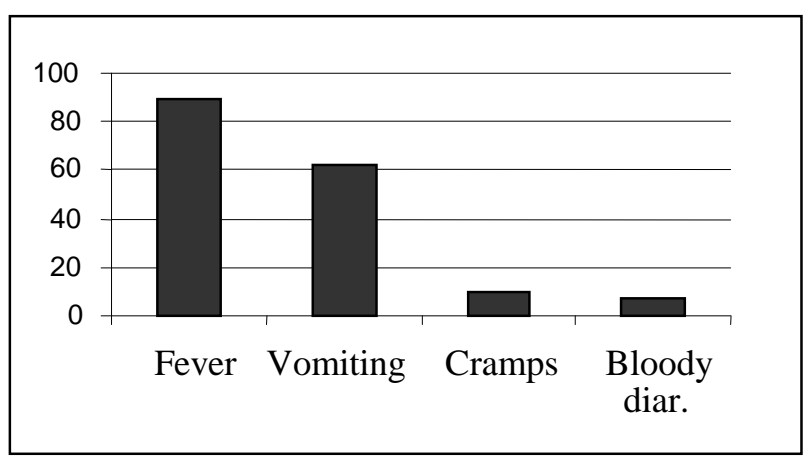

Fig. 1: Clinical symptoms of Aeromonas associated diarrhea

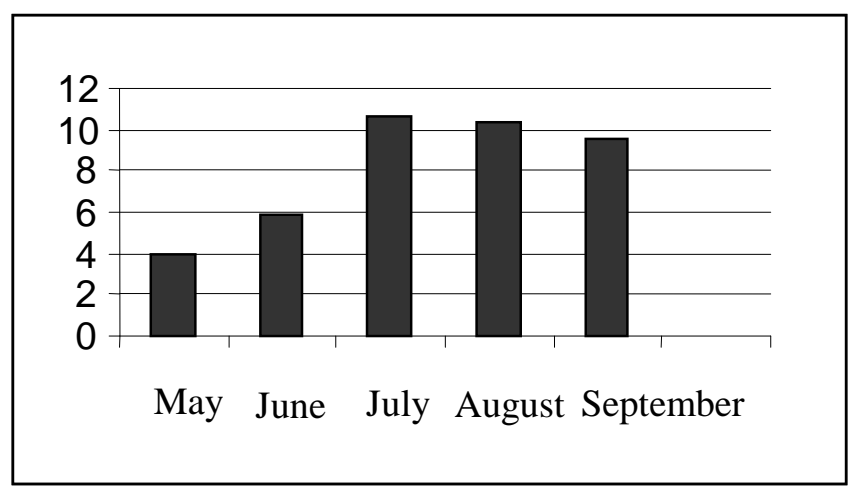

Fig. 2: The distribution of Aeromonas cases on summer months 


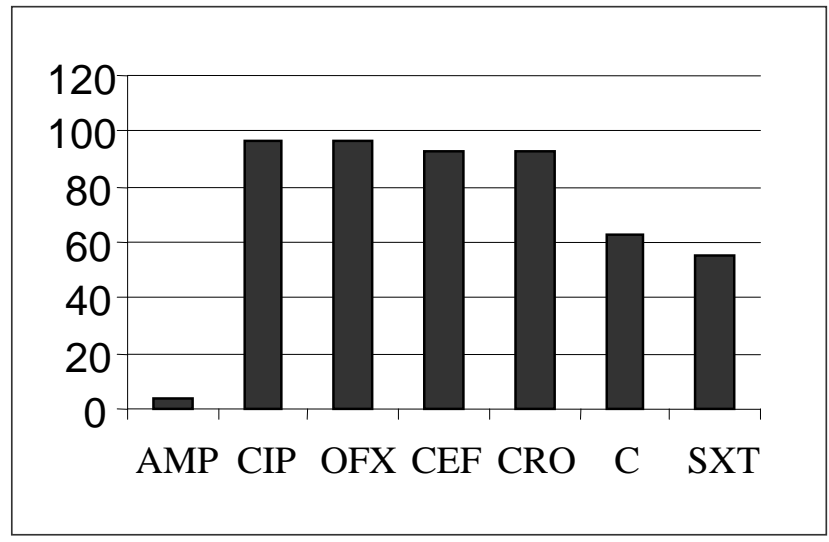

Fig. 3: Antibiotic susceptibility of the isolated Aeromonas strains

have been reported to be active against Aeromonas strains and it can be used as alternative therapy to quinolones.

The resistance to Ampicillin may be considered as a character to Aeromonas species as reported by many authors. ${ }^{13 \& 14}$ It was $96.6 \%$ in this study.

\section{REFRENCES}

1- P. R. Murray, E. J. Baron, M. A. Pfaller, F. C. Tenover and R. H. Yolken, "American Society for Microbiology", Washington (1999).

2- S. Holmberg, W. Schell, G. Fanning, et al., Ann. Intern. Med., 105 (1998).

3- M. J. Albert, M. Ansauzzaman, K. A. Talukder, A. K. Chopra, I. Kuhn, M. Rahman, et al. J. Clin. Microbiol., 38, 3785 (2000).
4- E. Gonzalez-Barca, C. J. Ardanuy, Carratala, A. Sanchez, A. Fernandez-Sevilla and A. Granena, Scand. J. Infect. Dis., 29, 91 (1997).

5- M. P. Grobush, K. Gobels, and D. Teichmann, Infection, 29, 109 (2001).

6- L. Vera, T. Sebastio, H. Sandra, C. Fabiana, C. Rosa and M. Debora, Rev. Microbiol., 29, 3704 (1998).

7- S. Massa, C. Altieri and A. angela, Int. J. Food Microbiol., 63, 169 (2001).

8- A. G. Komathi, S. Ananthan and S. Awand, Ind. J. Med. Res., 107, 252 (1998).

9- T. Teka, A. S. G. Faruque, M. I. Hossain and J. F. George, Annals of Tropical Pediatrics, 19, 15 (1999).

10- S. Siu-Wa and K. Cheung, J. Emeng. Infect. Dis., 10 (2003). 
11- J. Vila, R. Joaquin, G. Francisco, V. Martha, S. Lara, J. F. Maria and G. Joaquin, ibid., 9 (2003).

12- W. C. Ko, H. C. Lee, Y. C. Chuang, C. C. Liu and J. J. We, J. Infect., 40, 267 (2000).

13- L. O. Timothy and J. M. Janda, J. Clin. Microbiol., 37, 706 (1998).

14- W. C. Ko, K. W. YU, C. Y. Liu, C. T. Huang, H. S. Heu and Y. C. Chuang, Antimicrob. Agents Chemother., 40,1260 (1996).

15- S. Sinha, T. Shimada, T. Ramamurthy, S. K. Bhattacharya, S. Yamasaki, Y. Takeda and G. Balakrish Nair, India. J. Med Microbiol., 53, 527 (2004).
16- National Committee for Clinical Laboratory Standards, Performance standards for antimicrobial susceptibility testing; ninth informational supplement. Wayne, Pennsyslvania: NCCLS; 1999: document M100-S9, Vol. 19, No. 1, Table 2I.

17- G. Pazzaglia, R. B. Sack, E. Salazar, et al., J. Clin. Microbiol., 29, 1151 (1999). 